\title{
Frequency-Domain Hammerstein Self-Interference Canceller for In-Band Full-Duplex OFDM Systems
}

\author{
Kazuki Komatsu, Yuichi Miyaji, and Hideyuki Uehara \\ Department of Electrical and Electronic Information Engineering, Toyohashi University of Technology, Japan \\ Email: komatsu@comm.ee.tut.ac.jp, miyaji@ee.tut.ac.jp, uehara@tut.jp
}

\begin{abstract}
In-band full-duplex communications have been spotlighted because they can double the spectral efficiency of the current wireless communication systems. However, it is necessary to mitigate the self-interference (SI). Currently, several time-domain and frequency-domain SI cancellers have been proposed. Timedomain SI cancellers are based on the parallel Hammerstein (PH) model, and they have good flexibility with high computational cost. In contrast, frequency-domain SI cancellers can achieve high cancellation performance with low computational cost but they have less flexibility than time-domain PH based SI cancellers. In this paper, we propose a frequency-domain SI canceller based on the PH model. The proposed scheme estimates the frequency response of the SI channel and regenerates SI signals by the overlap-save method. Therefore, the computational complexity of the proposed scheme is less than time-domain PH based SI canceller. The performance of the proposed scheme is assessed by equivalent baseband signal simulations of a fullduplex transceiver. As a result, the proposed scheme achieves high SI cancellation as the time-domain PH based SI canceller with low computational cost. In addition, the convergence performance of the proposed scheme is faster than the time-domain scheme.
\end{abstract}

\section{INTRODUCTION}

In-band full duplex (IBFD) communications attract research attention because they are expected to improve the spectral efficiency compared to conventional half-duplex systems. However, self-interference (SI) are caused by simultaneous transmission and reception at the same frequency and limits the channel capacity [1]. On IBFD, it is necessary to reduce the power of the SI signal to the noise floor to demodulate the desired signal.

Recently, some IBFD systems and techniques have been researched [1]-[14]. A received SI signal is canceled by a radio-frequency (RF) analog SI canceller. The balun canceller [3] reduces a line of sight (LOS) component of the SI signal, and the multi-tap RF canceller [4] can reduce several delayed SI signals. Digital SI cancellers are necessary to eliminate long delayed and remained SI signals since RF cancellers cannot reduce the power of the SI signal to the noise floor. Digital SI cancellation algorithms can be classified into three categories: time-domain algorithms, frequency-domain algorithms, and reference receiver based algorithms. Time-domain digital SI cancellation algorithms [6]-[9] assume the SI channel including a transceiver path to be the parallel Hammerstein model, and they have good flexibility. For example, they can be trained by basic adaptive filters and choose components of canceled SI signal by changing basis functions. However, least mean squares (LMS) and recursive least squares learninig methods for them need computational cost of $\mathcal{O}(P N)$ and $\mathcal{O}\left(P^{2} N^{2}\right)$, respectively, and it is high for mobile terminals, where $P$ is the number of their basis functions, and $N$ is the number of taps of each branched FIR filter. Frequency-domain digital SI cancellation algorithms [3], [10], [11] have less flexibility than the Hammerstein based time-domain canceller. They estimate frequency responses of the SI channel in frequency-domain, and it needs low computational cost by using fast Fourier trasnform (FFT). The other category is the SI canceller with a reference receiver [12], [13]. They achieve high SI cancellation with simple algorithms but they need an additional auxiliary receiver.

In this paper, we propose a frequency-domain SI cancellation algorithm based on the parallel Hammerstein model. In a training period, it transmits appropriate symbols and estimates frequency responses of the SI channel in frequency-domain. Then, the SI-canceled signal is computed by the overlap-save method. The proposed scheme achieves high SI cancellation with low computational cost and good flexibility like timedomain cancellers.

\section{Self-Interference Signal Model}

The structure of a transceiver subject to this study is presented in Fig. 1. In this section, we derive the SI signal model on the transceiver. The transceiver consists of a transmitter and a receiver which have RF components such as IQ mixers, filters, and amplifiers. The method of the transceiver is orthogonalized frequency division multiplexing (OFDM). The isolation between a transmit antenna and a receive antenna is achieved by a passive antenna isolation, and a simple RF SI canceller which cancels only LOS SI signal is used. For simplicity, desired signals are not received, and both RF filters and variable gain amplifiers (VGA) of the transceiver have ideal characteristics.

\section{A. IQ Mixer}

The transmission baseband signal $x[n]$ is converted to an analog baseband signal by digital to analog converter (DAC), and it is up-converted by an IQ mixer. On an ideal IQ mixer, equivalent baseband signal of the output signal from the IQ mixer is equal to the input signal of the IQ mixer. But in fact, that is not satisfied because an actual IQ mixer has imbalances between I- and Q-phase carrier signals. Thus, the output signal of the transmitter IQ mixer can be expressed as

$$
x_{\mathrm{IQ}}(t)=g_{1}^{\mathrm{TX}}(\tau) * x(t)+g_{2}^{\mathrm{TX}}(\tau) * x^{*}(t)
$$

The final version of record is available at http://dx.doi.org/10.1109/WCNC.2017.7925765

(C) 2017 IEEE. Personal use of this material is permitted. Permission from IEEE must be obtained for all other uses, in any current or future media, including reprinting/republishing this material for advertising or promotional purposes, creating new collective works, for resale or redistribution to servers or lists, or reuse of any copyrighted component of this work in other works. 


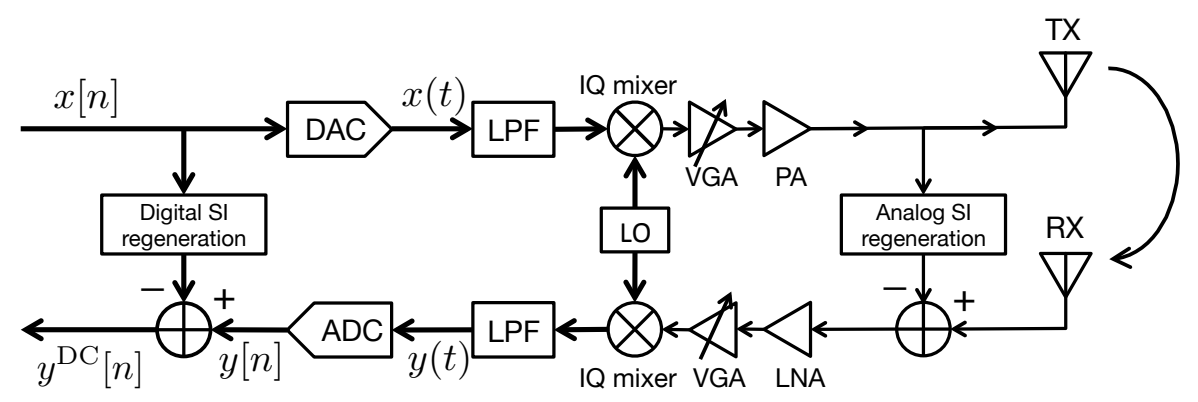

Fig. 1. A model of the assumed full duplex transceiver.

where $g_{1}^{\mathrm{TX}}(\tau)$ and $g_{2}^{\mathrm{TX}}(\tau)$ are I- and Q-phase impulse responses of the transmitter IQ mixer [15], and the binary operator $f * g$ denotes a convolution of $f$ and $g$. An indicator of the imbalance is called image rejection rate (IRR), and it is defined as

$$
\operatorname{IRR}^{\mathrm{TX}}(f)=\frac{\left|G_{1}^{\mathrm{TX}}(f)\right|^{2}}{\left|G_{2}^{\mathrm{TX}}(f)\right|^{2}},
$$

where $G_{1}^{\mathrm{TX}}(f)$ and $G_{2}^{\mathrm{RX}}(f)$ are frequency responses of $g_{1}^{\mathrm{TX}}(t)$ and $g_{2}^{\mathrm{TX}}(t)$, respectively.

On the assumption that $G_{1}^{\mathrm{TX}}(f)$ and $G_{2}^{\mathrm{TX}}(f)$ are constant at a whole band, The output signal of the transmitter IQ mixer can be simplified to

$$
x_{\mathrm{IQ}}(t)=x(t)+\frac{x^{*}(t)}{\mathrm{IRR}^{\mathrm{TX}}} .
$$

On the same assumption, the output signal of the receiver IQ mixer can be written by

$$
y_{\mathrm{IQ}}(t)=y_{\mathrm{VGA}}(t)+\frac{y_{\mathrm{VGA}}^{*}(t)}{\operatorname{IRR}^{\mathrm{RX}}}
$$

where $\operatorname{IRR}^{\mathrm{RX}}$ is an IRR of the receiver IQ mixer, and $y_{\mathrm{VGA}}(t)$ is the output signal of the receiver VGA.

\section{B. Power Amplifier}

The output signal of the transmitter IQ mixer $x_{\mathrm{IQ}}(t)$ is amplified by the power amplifier (PA) before transmitting from the antenna. Practical characteristics of a PA can be assumed to be linear at low output power, but the distorted output signal cannot be ignored for SI cancellers at high output power. The output signal at the PA is expressed as

$$
\begin{aligned}
x_{\mathrm{PA}}(t) & =f_{\mathrm{PA}}(\tau) *\left(a_{1} x_{\mathrm{IQ}}(t)+x_{\mathrm{IMD}}(t)\right), \\
x_{\mathrm{IMD}}(t) & =a_{3} \psi_{3}\left(x_{\mathrm{IQ}}(t)\right)+a_{5} \psi_{5}\left(x_{\mathrm{IQ}}(t)\right)+\cdots, \\
\psi_{p}(x) & =x|x|^{p-1},
\end{aligned}
$$

where $f_{\mathrm{PA}}(\tau)$ is an impulse response of the PA, and $x_{\mathrm{IMD}}(t)$ is an intermodulation distortion signal, and $a_{p}$ is a gain of a distorted signal $\psi_{p}$.

The indicator of the nonlinearity is called input intercept point, $\mathrm{IIP}_{p}$, or output intercept point, $\mathrm{OIP}_{p}$. When the power of the linear output signal is equal to the power of the third distorted output signal, the power of the input signal is equal to $\mathrm{IIP}_{3}$, and the power of the output signal is equal to $\mathrm{OIP}_{3}$.

\section{Self-Interference Coupling Channel}

The received SI signal at the receive antenna can be written by

$$
y_{\mathrm{ANT}}(t)=h(\tau) * x_{\mathrm{PA}}(t)+n_{\mathrm{th}}(t),
$$

where $h(\tau)$ is an impulse response of the SI channel and $n_{\text {th }}(t)$ is a thermal noise. The SI channel is strongly coupling, and a LOS wave is stronger than multi-path waves because the receive antenna is placed near the transmit antenna. Thus the characteristic of the SI channel is modeled to Rician fading channel, and its parameter is $K=25-40 \mathrm{~dB}$ when the distance between the transmit antenna and the receive antenna is less than $0.5 \mathrm{~m} \mathrm{[16].} \mathrm{The} \mathrm{isolation} \mathrm{between} \mathrm{the}$ transmit antenna and the receive antenna can be higher by using directional antennas and absorbers [17].

\section{Received Self-Inteference signal}

Taking RF impairments into account, the quantized received signal is written as

$$
y=\sum_{p: \text { odd }}\left\{h_{p} * \psi_{p}(x)+h_{p}^{\prime} * \psi_{p}^{*}(x)\right\}+z,
$$

where $h_{p}$ and $h_{p}^{\prime}$ are impulse responses of the SI channel, and $z$ is a total noise at the receiver. The received SI signal at frequency-domain is expressed as

$$
Y=\sum_{p: \text { odd }}\left\{H_{p} \Psi_{p}+H_{p}^{\prime} \Psi_{p}^{\prime}\right\}+Z,
$$

where $\Psi_{p}$ is a frequency-domain symbol of the $p$-th distorted transmitted signal, and $\Psi_{p}^{\prime}$ is an image signal of $\Psi_{p}$. In addition, $H_{p}$ and $H_{p}^{\prime}$ are frequency responses of $\Psi_{p}$ and $\Psi_{p}^{\prime}$, respectively. Thus, the received frequency-domain signal $Y$ can be described as a linear combination of $\Psi_{p}$ and $\Psi_{p}^{\prime}$.

\section{Proposed Frequency-Domain CANCEller Based on Parallel Hammerstein Model}

In this section, we describe the proposed frequency-domain SI canceller based on the parallel Hammerstein model as shown in Fig. 2. The frequency responses $H_{p}$ and $H_{p}^{\prime}$ are estimated in the training period of the proposed scheme. In the canceling period, the received SI signal is regenerated by the overlap-save method with $H_{p}$ and $H_{p}^{\prime}$. 


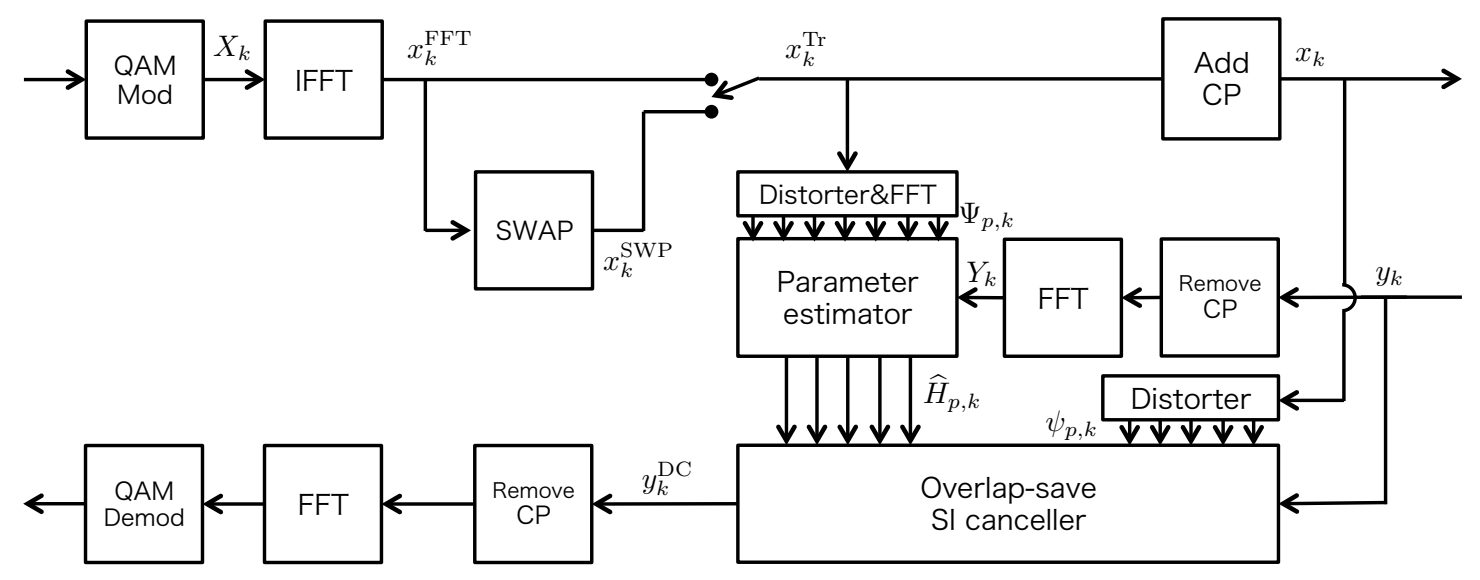

Fig. 2. Proposed SI canceller.

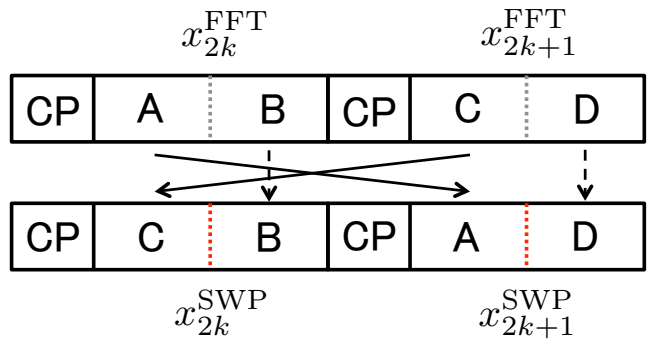

Fig. 3. Swapped training OFDM symbols.

\section{A. Generating Training Symbols}

In the training period of the proposed scheme, the transmission symbol, $x_{k}$, is manipulated from a pure OFDM symbol to utilize effects of the cyclic prefix (CP) as shown in Fig. 3. The swapping manipulation is necessary to achieve high SI cancellation, because there are no discontinuities in an OFDM symbol, and they appear between symbols. Additionally, $H_{p, k}$, which is the frequency response of $\psi_{p}\left(x_{k}\right)$, can be estimated with high accuracy by the CP.

The first step of generating training symbols is to modulate training bits to $X_{k}$ by QAM and to convert them to a timedomain symbol $x_{k}^{\mathrm{FFT}}$, such that

$$
x_{k}^{\mathrm{FFT}}=\operatorname{IFFT}\left\{X_{k}\right\},
$$

where the operator IFFT $\{\cdot\}$ is the inverse fast Fourier transform operator.

In the second step of generating, the first half of $x_{2 k}^{\mathrm{FFT}}$ is swapped to the first half of $x_{2 k+1}^{\mathrm{FFT}}$, such that

$$
\begin{aligned}
x_{2 k}^{\mathrm{SWP}} & =\left\{x_{2 k+1,\left[0 \ldots N_{\mathrm{FFT}} / 2\right]}^{\mathrm{FFT}}, x_{2 k,\left[N_{\mathrm{FFT}} / 2 \ldots N_{\mathrm{FFT}}\right]}^{\mathrm{FFT}},\right. \\
x_{2 k+1}^{\mathrm{SWP}} & =\left\{x_{2 k,\left[0 \ldots N_{\mathrm{FFT}} / 2\right]}^{\mathrm{FFT}}, x_{2 k+1,\left[N_{\mathrm{FFT}} / 2 \ldots N_{\mathrm{FFT}}\right]}^{\mathrm{FFT}},\right.
\end{aligned}
$$

where $N_{\text {FFT }}$ is the FFT size of OFDM, and $s_{[n \ldots m]}$ is equal to $\{s[n], s[n+1], \cdots, s[m-1]\}$. By this manipulation, discontinuities are produced in OFDM symbols without destructing the CP structure, and the parameter estimator of the proposed method can estimate discontinuities of the SI signal with high accuracy. Furthermore, the number of swapped OFDM training symbols can be less than pure OFDM training symbols because side lobes increased by discontinuities become the minority in all power of the SI signal.

In the final step of generating training symbols, $x_{k}^{\mathrm{SWP}}$ is used $N_{\text {SWP }}$ times as a transmission symbol $x_{k}^{\text {Tr }}$, and pure OFDM symbol $x_{k}^{\mathrm{FFT}}$ is used as a transmission signal after transmitting $x_{k}^{\text {SWP }}$ as written by

$$
x_{k}^{\mathrm{Tr}}= \begin{cases}x_{k}^{\mathrm{SWP}} & \text { for } k<N_{\mathrm{SWP}}, \\ x_{k}^{\mathrm{FFT}} & \text { for } k \geq N_{\mathrm{SWP}} .\end{cases}
$$

Then, the transmission symbols $x_{k}^{\mathrm{Tr}}$ and $N_{\mathrm{CP}}$-sized $\mathrm{CP}$ are jointed as (13) and transmitted.

$$
\begin{aligned}
x_{k}[n] & =\operatorname{AddCP}\left\{x_{k}^{\operatorname{Tr}}\right\} . \\
\operatorname{AddCP}\{z\} & =\left\{z_{\left[N_{\mathrm{FFT}}-N_{\mathrm{CP}} \ldots N_{\mathrm{FFT}}\right]}, z\right\} .
\end{aligned}
$$

\section{B. Frequency-Domain Training}

On the proposed method, the frequency response of the SI channel can be estimated by well-known adaptive algorithms in frequency-domain scheme, and they estimate the frequency response of the SI channel at each discrete frequency, $f$, independently. Least squares (LS), recursive LS (RLS) and normalized least mean squares (NLMS) algorithms applied to the proposed scheme are shown in the following.

\section{1) Least squares parameter estimation:}

LS algorithm is one of the most fundamental parameter estimation algorithms. At digital baseband, the received signal can be expressed as

$$
\begin{aligned}
Y_{k}[f] & =\operatorname{FFT}\left\{y_{k,\left[N_{\mathrm{CP}} \ldots N_{\mathrm{CP}}+N_{\mathrm{FFT}}\right]}[f]\right. \\
& =H_{1, k}[f] \Psi_{1, k}[f]+H_{2, k}[f] \Psi_{2, k}[f] \\
& +\cdots+H_{P, k}[f] \Psi_{P, k}[f]+Z_{k}[f], \\
\Psi_{p, k} & = \begin{cases}\operatorname{FFT}\left\{\psi_{p}\left(x_{k}^{\operatorname{Tr}}\right)\right\}, & \text { for odd } p, \\
\operatorname{FFT}\left\{\psi_{p-1}^{*}\left(x_{k}^{\mathrm{Tr}}\right)\right\}, & \text { for even } p,\end{cases}
\end{aligned}
$$

where $y_{k}$ is the $k$-th received symbol and $Z_{k}[f]$ is total noise at the receiver. 
To apply LS algorithm for estimating the frequency response, $H_{p, k}[f]$ is assumed to be static and equal to $H_{p}[f]$. Additonally, we introduce vector-matrix notations, $\mathbf{Y}[f], \mathbf{\Psi}[f]$ and $\mathbf{H}[f]$, such that

$$
\begin{aligned}
& \mathbf{Y}[f]=\left[Y_{0}[f] Y_{1}[f] \cdots Y_{N_{\mathrm{Tr}}-1}[f]\right]^{T}, \\
& \boldsymbol{\Psi}[f]=\left[\begin{array}{cccc}
\Psi_{1,0}[f] & \Psi_{2,0}[f] & \cdots & \Psi_{P, 0}[f] \\
\Psi_{1,1}[f] & \Psi_{2,1}[f] & \cdots & \Psi_{P, 1}[f] \\
\vdots & \vdots & \ddots & \vdots \\
\Psi_{1, N_{\mathrm{Tr}}-1}[f] & \Psi_{2, N_{\mathrm{Tr}}-1}[f] & \cdots & \Psi_{P, N_{\mathrm{Tr}}-1}[f]
\end{array}\right] \\
& \mathbf{H}[f]=\left[H_{1}[f] \cdots H_{p}[f] \cdots H_{P}[f]\right]^{T},
\end{aligned}
$$

where $N_{\operatorname{Tr}}$ is the number of training symbols, and $P$ is the number of basis functions. By using vector-matrix notations, $\mathbf{Y}[f]$ can be expressed as

$$
\mathbf{Y}[f]=\mathbf{\Psi}[f] \mathbf{H}[f]+\mathbf{Z}[f],
$$

where $\mathbf{Z}[f]$ consists of $Z_{k}[f]$. Then, the LS-estimated frequency responses are expressed as

$$
\begin{aligned}
\widehat{\mathbf{H}}[f] & =\left[\widehat{H}_{1}[f] \widehat{H}_{2}[f] \cdots \widehat{H}_{P}[f]\right]^{T} \\
& =\left(\boldsymbol{\Psi}^{H}[f] \mathbf{\Psi}[f]\right)^{-1} \boldsymbol{\Psi}^{H}[f] \mathbf{Y}[f] .
\end{aligned}
$$

2) Recursive least squares parameter estimation:

LS algorithm requires matrix inversion or singular value decomposition (SVD). On the other hand, recursive LS algorithm can recursively estimate parameters which minimize least square errors without matrix inversion and SVD. On the proposed scheme, RLS algorithm is expressed as

$$
\begin{aligned}
\mathbf{\Psi}_{k}[f] & =\left[\Psi_{1, k}[f] \Psi_{2, k}[f] \cdots \Psi_{P, k}[f]\right]^{T}, \\
\widehat{\mathbf{H}}_{k}[f] & =\widehat{\mathbf{H}}_{k-1}[f]+E_{k}[f] \mathbf{G}_{k}[f], \\
E_{k}[f] & =Y_{k}[f]-\mathbf{\Psi}_{k}^{T}[f] \mathbf{H}_{k-1}[f], \\
\mathbf{G}_{k}[f] & =\frac{\mathbf{P}_{k-1}[f] \mathbf{\Psi}_{k}^{*}[f]}{\lambda+\mathbf{\Psi}_{k}^{T}[f] \mathbf{P}_{k-1}[f] \mathbf{\Psi}_{k}^{*}[f]}, \\
\mathbf{P}_{k}[f] & =\lambda^{-1}\left\{\mathbf{P}_{k-1}[f]-\mathbf{G}_{k}[f] \mathbf{\Psi}_{k}^{T}[f] \mathbf{P}_{k-1}[f]\right\},
\end{aligned}
$$

where $\boldsymbol{\Psi}_{k}[f]$ is the $k$-th input signal vector of an RLS adaptive filter at a discrete frequency, $f$, and $\widehat{\mathbf{H}}_{k}[f]$ is the $k$-th estimated frequency response at $f$, and $\lambda$ is called forgetting factor. Furthermore, $\widehat{\mathbf{H}}_{k}[f]$ and $\mathbf{P}_{k}[f]$ are initialized to $\mathbf{0}$ and $\delta^{-1} \mathbf{I}$ where $\delta$ is very small positive value. The total number of multiplications of complex value for computing (23)-(27) is $4 P^{2}+4 P$.

3) Normalized least mean squares parameter estimation:

NLMS parameter estimation algorithm has much lower computation cost and complexity than RLS algorithm. NLMS algorithm is expressed as

$$
\begin{aligned}
\widehat{\mathbf{H}}_{k}[f] & =\widehat{\mathbf{H}}_{k-1}[f]+\frac{\mu}{Q_{k}[f]} E_{k}[f] \mathbf{\Psi}_{k}^{*}[f], \\
E_{k}[f] & =Y_{k}[f]-\boldsymbol{\Psi}_{k}^{T}[f] \mathbf{H}_{k-1}[f], \\
Q_{k}[f] & =(1-\alpha) Q_{k-1}[f]+\alpha \boldsymbol{\Psi}_{k}^{H}[f] \boldsymbol{\Psi}_{k}[f],
\end{aligned}
$$

where $\mu$ and $\alpha$ are positive constant values, and $\boldsymbol{\Psi}_{k}[f]$ and $\widehat{\mathbf{H}}_{k}[f]$ are the same values as for RLS. The total number of multiplications of complex values for computing (28)-(30) is $2 P$, and it is $N$ times faster than the time-domain NLMS, where $N$ is the number of taps of a time-domain FIR filter.

\section{Time-Domain Canceling Using Frequency Response}

In this section, we describe the overlap-save method for the proposed scheme on time-domain using $\mathbf{H}_{p, k}[f]$ without conversion to impulse responses. When a signal $x$ is periodic, $(h * x)$ is known as a circular convolution and it can be computed efficiently with FFT algorithm by IFFT $\{$ FFT $\{x\}$ FFT $\{h\}\}$. In general, this algorithm is not used to regenerating SI signals because the transmitted signal is not periodic. For this reason, SI signals are regenerated by the overlap-save method [18] expressed as

$$
\begin{aligned}
& \psi_{p, i}^{\mathrm{RG}}[n]=\operatorname{IFFT}_{2 N_{\mathrm{FFT}}}\left\{\Psi_{p, i}^{\mathrm{RG}}\right\}\left[N_{\mathrm{FFT}}-N_{\mathrm{CP}}+n\right], \\
& \Psi_{p, i}^{\mathrm{RG}}= \begin{cases}H_{p, k} \mathrm{FFT}_{2 N_{\mathrm{FFT}}}\left\{\psi_{p}\left(x_{i}^{c}\right)\right\}, & \text { for odd } p, \\
H_{p, k} \mathrm{FFT}_{2 N_{\mathrm{FFT}}}\left\{\psi_{p-1}^{*}\left(x_{i}^{c}\right)\right\} & \text { for even } p,\end{cases} \\
& x_{i}^{c}=\left\{x_{i-1,\left[2 N_{\mathrm{CP}} \ldots N_{\mathrm{CP}}+N_{\mathrm{FFT}}\right]}, x_{i}\right\}
\end{aligned}
$$

where $\psi_{p, i}^{\mathrm{RG}}[n]$ is the $p$-th distorted $i$-th regenerated OFDM symbol of the SI signal, and $\mathrm{FFT}_{M}\{\cdot\}, \operatorname{IFFT}_{M}\{\cdot\}$ are $M$ sized FFT and inverse FFT, respectively. Finally, the digital SI canceled signal can be written by

$$
y_{i}^{\mathrm{DC}}[n]=y_{i}[n]-\sum_{p=1}^{P} \psi_{p, i}^{\mathrm{RG}}[n] .
$$

\section{Computational Cost and Complexity}

We describe the derivation process of computational cost and complexity of the proposed scheme in the following.

In the training period of the proposed scheme, received OFDM symbols are decomposed to discrete frequency components by FFT after removing CPs. This process requires $\frac{1}{2} N_{\mathrm{FFT}} \log _{2} N_{\mathrm{FFT}}$ times multiplications of two complex numbers per symbol. The transmitted symbols before adding CPs are distorted by $\psi_{p}(\cdot)$ and also decomposed to discrete frequency components by FFT, where $\psi_{p}(\cdot)$ can be computed a priori and implemented by a lookup table. The total computational cost of transforming received and transmitted symbols to frequency-domain is $\frac{1}{4}(P+2) N_{\text {FFT }} \log _{2} N_{\text {FFT }}$ per symbol because $\operatorname{FFT}\left\{\psi_{p}^{*}(x)\right\}$ can be computed by frequency-inversion and conjugation of FFT $\left\{\psi_{p}(x)\right\}$. In the next step of the training period, the proposed scheme estimates $H_{p, k}[f]$ from $Y_{k}[f]$ and $\Psi_{p, k}[f]$. NLMS algorithm and RLS algorithm require $2 P$ and $4 P^{2}+4 P$ complex multiplications per discrete frequency per symbol, respectively. Thus, the whole computational cost of the training period is $\frac{1}{4}(P+2) N_{\mathrm{FFT}} \log _{2} N_{\mathrm{FFT}}+A N_{\mathrm{FFT}}$ per symbol, where $A$ is $2 P$ (NLMS used) or $4 P^{2}+4 P$ (RLS used).

In the canceling period, the overlap-save method is used $P$ times on the proposed scheme. By the same trivial technique for computing FFT $\left\{\psi_{p}^{*}(x)\right\}$, this process requires $\frac{3}{2} P N_{\mathrm{FFT}}\left(\log _{2} N_{\mathrm{FFT}}+1\right)$ complex-multiplications per symbol since one time overlap-save method can be computed by

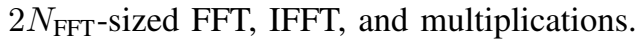


TABLE I

Simulation SPECIFICATIONS

\begin{tabular}{c|c}
\hline \hline Parameter & Value \\
\hline Oversampling rate & 4 \\
\hline Modulation & OFDM \\
\hline Constellation & 16QAM \\
\hline Size of FFT $N_{\mathrm{FFT}}$ & 256 \\
\hline Number of subcarriers & 52 \\
\hline Cyclic prefix $N_{\mathrm{CP}}$ & 80M samples $/ \mathrm{sec}$ \\
\hline Sampling rate & Uniform-random data \\
\hline Transmit data & Rayleigh fading \\
\hline SI channel after RF-SIC & 64 samples \\
\hline Channel order & $15 \mathrm{dBm}$ \\
\hline Transmit power & varied \\
\hline IRR & $17 \mathrm{dBm}$ \\
\hline Highest order of IMD & $27 \mathrm{~dB}$ \\
\hline IIP3 & $4 \mathrm{~dB}$ \\
\hline PA Gain & $14 \mathrm{bits}$ \\
\hline Noise figure of LNA & $\left\{x, x^{*}, x|x|^{2}, x^{*}|x|^{2}, x|x|^{4}, x^{*}|x|^{4}\right\}$ \\
\hline Number of ADC bits
\end{tabular}

\section{Performance Simulations}

In this section, we evaluate the performance of the proposed scheme by equivalent baseband signal simulations of a full-duplex transceiver as shown in Fig. 1. The simulation parameters are presented as TABLE I, which are based on [7]. In this simulations, the dynamic range of the receiver ADC is about $79 \mathrm{~dB}$ [19]. The imbalance of IQ mixers is realized by adding an image signal, and the coefficients of nonlinearity of the PA are predetermined based on IIP. The parameters of both RLS algorithm and LMS algorithm are set to the optimal coefficients which can achieve the best steady-state cancellation performance in each canceller. This scenario ensures that the results show the true performance of each canceller. Additionally, in this simulations, the SI cancellation rate is defined as

$$
\text { Cancellation }=\frac{\mathbb{E}\left[|y|^{2}\right]}{\mathbb{E}\left[\left|y^{\mathrm{DC}}\right|^{2}\right]},
$$

where $\mathbb{E}[s]$ denotes the expected value of $s$.

\section{A. Cancellation Performance}

Fig. 4 shows the cancellation performance of both the proposed frequency-domain parallel Hammerstein based method (FH) and time-domain parallel Hammerstein based method (PH) [8]. In addition, the parameters of both cancellers are optimized by LS algorithm, and the self-interference and noise power ratio (INR) varies from $20 \mathrm{~dB}$ to $80 \mathrm{~dB}$, where INR is defined as

$$
\mathrm{INR}=\frac{\mathbb{E}\left[G_{\mathrm{LNA}}^{2}\left|\left(h(\tau)-h_{\mathrm{RF}}(\tau)\right) * x_{\mathrm{PA}}(t)\right|^{2}\right]}{\mathbb{E}\left[\left|n_{\mathrm{LNA}}(t)\right|^{2}\right]},
$$

where $h_{\mathrm{RF}}(\tau)$ is the impulse response of the RF SI canceller, and $G_{\mathrm{LNA}}$ is the gain of the receiver LNA, and $n_{\mathrm{LNA}}(t)$ is a total noise of the output signal of the receiver LNA. In the situation of Fig. 4, the number of training symbols, $N_{\mathrm{Tr}}$, is
60 and swapped trainning symbols, $N_{\mathrm{SWP}}$, is $\infty$, and both SI cancellers can be assumed to be trained completely. The cancellation performance of the proposed scheme is similar to the time-domain method, and they achieve perfect cancellation of the SI signal when INR is below $60 \mathrm{~dB}$ even under worse IRR. Thus, the proposed method has a comprehensive cancellation capability as same as the time-domain method. When INR is higher than $70 \mathrm{~dB}$, SI cancellation is saturated because linear combinations of the basis functions of TABLE I cannot represent the received SI signal completely. The complete set of basis functions are discussed in [20], and in our future work, we apply them to the proposed method.

In Fig. 5, the convergence of both cancellers with NLMS and RLS algorithm is shown at INR $=50 \mathrm{~dB}$ and $N_{\mathrm{SWP}}=\infty$. The proposed scheme with RLS algorithm achieves about $49 \mathrm{~dB}$ SI cancellation with three or four training symbols, and the time-domain scheme achieves about $49 \mathrm{~dB}$ SI cancellation with 28 training symbols. Hence, convergence speed of the frequency-domain scheme with RLS algorithm is faster than the time-domain scheme because the time-domain signals are strongly colored. Generally, convergence speed of an adaptive algorithm decreases with colored input signals. On the other hand, the input signal of the RLS adaptive filter of the frequency-domain scheme has flat spectrum and weak correlation between their elements. For this reason, the timedomain scheme needs nine times more training symbols than the frequency-domain scheme. When NLMS algorithm is used, the frequency-domain scheme provides about $49 \mathrm{~dB}$ SI cancellation with 38 training symbols, but the time-domain scheme cannot reach $40 \mathrm{~dB}$ for the first 60 symbols. This is due to the same reason for the RLS case.

Fig. 6 shows the convergence of the frequency-domain scheme with RLS algorithm when $N_{\text {SWP }}$ is changed from 0 to 4. The proposed scheme cannot achieve 50-dB SI cancellation at $N_{\mathrm{SWP}}<3$, and the result at $N_{\mathrm{SWP}}=4$ is similar to the case of $N_{\mathrm{SWP}}=\infty$ shown in Fig. 5. Consequently, the sufficient number of $N_{\text {SWP }}$ is four to achieve high SI cancellation.

\section{B. Computational Complexity and Cost}

TABLE II shows computational complexity and cost of the time-domain parallel Hammerstein based SI canceller and the proposed frequency-domain parallel Hammerstein based SI canceller when the parameters are the same as simulation evaluations. At deriving complexity, the number of basis functions, $P$, and the number of taps of a time-domain FIR filter, $N$, are assumed to be variables, and other parameters are treated as constant. Besides, the number of operations is calculated as multiplications of two complex numbers. The proposed scheme with RLS algorithm is about $4.02 \times 10^{3}$ times faster than the time-domain scheme with RLS algorithm and about 5.22 times faster than the time-domain scheme with NLMS algorithm at the training period. In addition, at the canceling period, the proposed scheme is about 5.93 times faster than the time-domain scheme. 


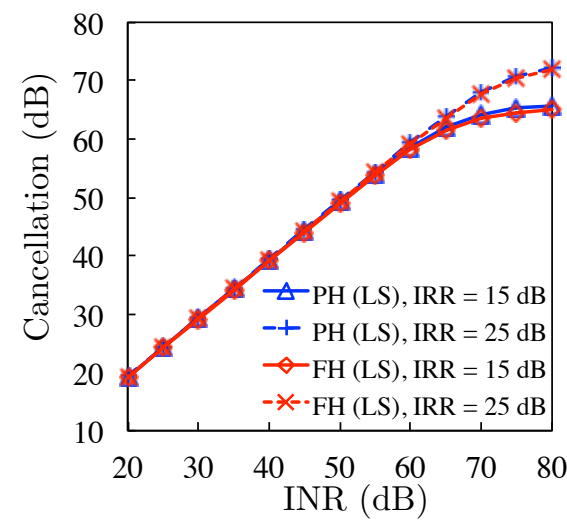

Fig. 4. The SI cancellation performance at $N_{\mathrm{Tr}}=60$.

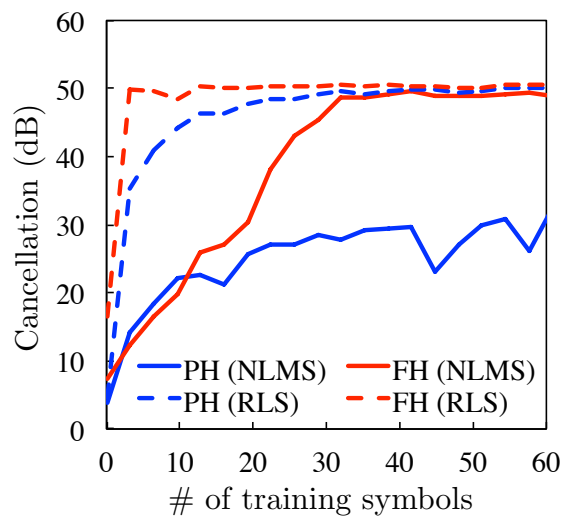

Fig. 5. The convergence of each canceller at $\mathrm{INR}=50 \mathrm{~dB}$ and IRR $=25 \mathrm{~dB}$.

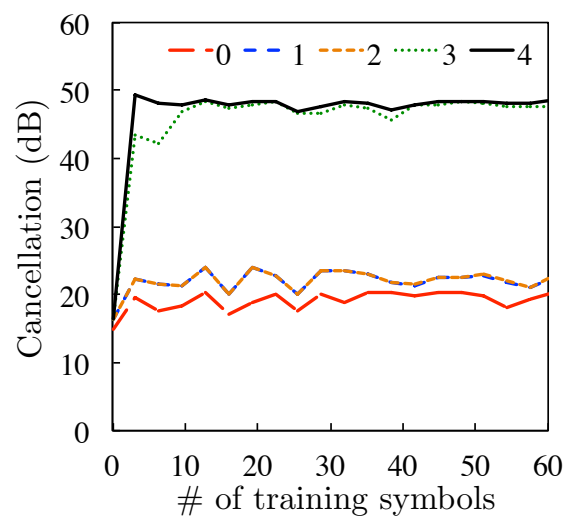

Fig. 6. The convergence performance of the proposed scheme with RLS algorithm at $N_{\text {SWP }}=0 \ldots 4$ and $\mathrm{IRR}=25 \mathrm{~dB}$.
TABLE II

COMPUTATIONAL COMPLEXITY AND COST PER SAMPLE

\begin{tabular}{l|cc|cc}
\hline \hline Canceller scheme & \multicolumn{2}{|c|}{ time-domain scheme } & \multicolumn{2}{c}{ proposed scheme } \\
\hline Adaptive algorithm & RLS & NLMS & RLS & NLMS \\
\hline Complexity of training & $\mathcal{O}\left(P^{2} N^{2}\right)$ & $\mathcal{O}(P N)$ & $\mathcal{O}\left(P^{2}\right)$ & $\mathcal{O}(P)$ \\
\hline \# of ops. of training & $591 \times 10^{3}$ & 768 & 147 & 22.4 \\
\hline Complexity of canceling & \multicolumn{2}{|c|}{$\mathcal{O}(P N)$} & \multicolumn{2}{c}{$\mathcal{O}(P)$} \\
\hline \# of ops. of canceling & \multicolumn{2}{|c|}{384} & \multicolumn{2}{c}{64.8} \\
\hline \hline
\end{tabular}

\section{CONCLUSION}

In this paper, we have proposed a frequency-domain digital self-interference canceller based on the parallel Hammerstein model. The proposed scheme decreases computational cost by estimating the characteristic of the SI channel on frequencydomain and using overlap-save method for regenerating SI signals. In addition, the proposed scheme has good compatibility with the time-domain parameter estimation algorithms such as least squares, recursive least squares, and normalized least mean squares algorithm. The performance of the proposed scheme is assessed by equivalent baseband signal simulations of a full-duplex transceiver. The results show that the proposed scheme achieves high SI cancellation as well as the timedomain scheme and fast convergence with low computational cost.

\section{REFERENCES}

[1] J. I. Choi, M. Jain, K. Srinivasan, P. Levis, and S. Katti, "Achieving Single Channel, Full Duplex Wireless Communication,” in Proc. ACM 16th Annual International Conference on Mobile Computing and Networking (MobiCom), pp. 1-12, Sept. 2010.

[2] D. Kim, H. Lee, and D. Hong, "A Survey of In-Band Full-Duplex Transmission: From the Perspective of PHY and MAC Layers," IEEE Communications Surveys \& Tutorials, vol. 17, no. 4, pp. 2017-2046, Feb. 2015.

[3] M. Jain, J. I. Choi, T. Kim, D. Bharadis, S. Seth, K. Srinivasan, P. Levis, S. Katti, and P. Sinha, "Practical, Real-Time, Full Duplex, Wireless," in Proc. ACM 17th MobiCom, pp. 301-312, Sept. 2011.

[4] J. Tamminen et al., "Digitally-Controlled RF Self-Interference Canceller for Full-Duplex Radios," in Proc. 24th European Signal Processing Conference (EUSIPCO), pp. 783-787, Aug. 2016.

[5] D. Bharadia, E. McMilin, and, S. Katti, "Full Duplex Radios," in Proc. ACM SIGCOMM 2013, pp. 475-386, Aug. 2013.

[6] L. Anttila, D. Korpi, V. Syrjälä, and M. Valkama, "Cancellation of Power Amplifier Induced Nonlinear Self-Interference in Full-Duplex Transceivers," in Proc. 2013 Asilomar Conference Signals, Systems and Computers, pp. 1-6, Nov. 2013.
[7] D. Korpi, L. Anttila, V. Syrjälä, and M. Valkama, "Widely-Linear Digital Self-Interference Cancellation in Direct-Conversion Full-Duplex Transceiver," IEEE Journals on Selected Areas in Communications, vol. 32, no. 9, pp. 1674-1687, Oct. 2014.

[8] M. Heino, D. Korpi, T. Huusari, E. Antonio-Rodríguez, S. Venkatasubramanian, T. Riihonen, L. Anttila, C. Icheln, K. Haneda, R. Wichman, and M. Valkama, "Recent Advances in Antenna Design and Interference Cancellation Algorithms for In-Band Full Duplex Relays," IEEE Communication Magazine, vol. 53, no. 5, pp. 91-101, May 2015.

[9] D. Korpi, Y.-S. Choi, T. Huusari, L. Anttila, S. Talwar, and M. Valkama, "Adaptive Nonlinear Digital Self-Interference Cancellation for Mobile Inband Full-Duplex Radio: Algorithms and RF Measurements," in Proc. IEEE Global Communications Conference (GLOBECOM), Dec. 2015.

[10] E. Ahmed, A. M. Eltawil, and A. Sabharwal, "Self-Interference Cancellation with Nonlinear Distortion Suppression for Full-Duplex Systems," in Proc. 2013 Asilomar Conference on Signals, Systems and Computers, Nov. 2013.

[11] E. Ahmed, A. M. Eltawil, and A. Sabharwal, "Self-Interference Cancellation with Phase Noise Induced ICI Suppress for Full Duplex Systems," in Proc. IEEE GLOBECOM, Dec. 2013.

[12] E. Ahmed and A. M. Eltawil, "All-Digital Self-Interference Cancellation Technique for Full-Duplex Systems," IEEE Transactions on Wireless Communications, vol. 14, no. 7, pp. 3519-3532, July 2015.

[13] D. Korpi, L. Anttila, and M. Valkama, "Reference Receiver Based Digital Self-Interference Cancellation in MIMO Full-Duplex Transceivers," in Proc. IEEE GLOBECOM Workshops, pp. 1001-1007, Dec. 2014.

[14] S. Li and R. D. Murch, "An Investigation Into Baseband Techniques for Single-Channel Full-Duplex Wireless Communication Systems," IEEE Transactions on Wireless Communications, vol. 13, no. 9, pp. 47944806, Sept. 2014.

[15] M. Valkama and M. Renfors, "Digital Filter Design for I/Q Imbalance Compensation," in Proc. 10th EUSIPCO, pp. 1-4, Sept. 2000.

[16] M. Duarte, C. Dick, and A. Sabharwal, "Experiment-Driven Characterization of Full-Duplex Wireless System," IEEE Transactions on Wireless Communications, vol. 11, no. 12, pp. 4296-4307, Dec. 2012.

[17] E. Everett, A. Sahai, and A. Sabharwal, "Passive Self-Interference Suppression for Full-Duplex Infrastructure Nodes," IEEE Transactions on Wireless Communucation, vol. 13, no. 2, pp. 680-694, Feb. 2014.

[18] W. H. Press, S. A. Teukolsky, W. T. Vetterling, B. P. Flannery, "Fourier and Spectral Applications," in Numerical Recipes The Art of Scientific Computing, third ed. Cambridge, UK: Cambridge Univ. Press, 2007, ch. 13, sec. 1, pp. 646-647.

[19] D. Korpi, T. Riihonen, V. Syrjälä, L. Anttila, and M. Valkama, "FullDuplex Transceiver System Calculations: Analysis of ADC and Linearity Challenges," IEEE Transactions on Wireless Communications, vol. 13, no. 7, pp. 3821-3836, July 2014 .

[20] D. Korpi, T. Huusari, Y.-S. Choi, L. Anttila, S. Talwar, and M. Valkama, "Digital Self-Interference Cancellation under Nonideal RF Components: Advanced Algorithms and Measured Performance," in Proc. IEEE 16th International Workshop on Signal Processing Advances in Wireless Communications (SPAWC), pp. 286-290 Jun. 2015. 